\title{
On the Diamond Bessel Klein Gordon operator related to linear differential equation
}

\section{Wanchak Satsanit}

Department of Mathematics, Faculty of Science, Maejo University, Chiang Mai, 50290, Thailand.

\begin{abstract} sense of Distribution theory considering the properties of the convolution of the Green function. Finally, we solve the following equation

$$
\left(\nabla_{\mathrm{B}}+\mathrm{d}^{2}\right)^{\mathrm{k}} \mathrm{u}(\mathrm{x})=\sum_{\mathrm{r}=0}^{\mathrm{m}} \mathrm{c}_{\mathrm{r}}\left(\nabla_{\mathrm{B}}+\mathrm{d}^{2}\right)^{\mathrm{k}} \delta .
$$

It was found that the type of above equation depend on the relationship between the value $\mathrm{k}$ and $\mathrm{m}$.

Keywords: Diamond Bessel operator, Diamond Klein Gordon Bessel operator, tempered distribution.

2010 MSC: 46F10, 46F12.
\end{abstract}

In this paper, first, we study the Green function of the Diamond Klein Gordon Bessel operator iterated k times. We give a

(C)2019 All rights reserved.

\section{Introduction}

The operator $\diamond^{k}$ has been first by Kananthai [3] and is named as the Diamond operator iterated $k$ times and defined by

$$
\diamond^{k}=\left(\left(\sum_{i=1}^{p} \frac{\partial^{2}}{\partial x_{i}^{2}}\right)^{2}-\left(\sum_{j=p+1}^{p+q} \frac{\partial^{2}}{\partial x_{j}^{2}}\right)^{2}\right)^{k}, p+q=n,
$$

where $\mathrm{n}$ is the dimension of the space $\mathbb{R}^{n}$, for $x=\left(x_{1}, x_{2}, \cdots, x_{n}\right) \in \mathbb{R}^{n}$ and $k$ is a nonnegative integer. The operator $\diamond^{k}$ can be expressed in the form

$$
\diamond^{k}=\triangle^{k} \square^{k}=\square^{k} \triangle^{k},
$$

where $\triangle^{k}$ is the Laplacian operator iterated $k$-times is defined by

$$
\Delta^{k}=\left(\frac{\partial^{2}}{\partial x_{1}^{2}}+\frac{\partial^{2}}{\partial x_{2}^{2}}+\cdots+\frac{\partial^{2}}{\partial x_{n}^{2}}\right)^{k},
$$

and $\square^{k}$ is the Ultrahyperbolic operator iterated k-times defined by

\footnotetext{
${ }^{*}$ Corresponding author

Email address: wanchack@gmail . com (Wanchak Satsanit)

doi: $10.22436 /$ jnsa.012.08.06
}

Received: 2017-02-19 Revised: 2019-02-27 Accepted: 2019-03-05 


$$
\square^{k}=\left(\frac{\partial^{2}}{\partial x_{1}^{2}}+\frac{\partial^{2}}{\partial x_{2}^{2}}+\cdots+\frac{\partial^{2}}{\partial x_{p}^{2}}-\frac{\partial^{2}}{\partial x_{p+1}^{2}}-\frac{\partial^{2}}{\partial x_{p+2}^{2}}-\cdots-\frac{\partial^{2}}{\partial x_{p+q}^{2}}\right)^{k} .
$$

Kananthai [3] has shown that the solution of the convolution form

$$
u(x)=(-1)^{\mathrm{k}} \mathrm{R}_{2 \mathrm{k}}^{e}(x) * \mathrm{R}_{2 \mathrm{k}}^{\mathrm{H}}(x),
$$

is a unique elementary solution of the operator $\diamond^{k}$, where $R_{2 k}^{e}(x)$ and $R_{2 k}^{H}(x)$ are defined by (2.4) and (2.2) with $\alpha=2 k$ respectively, that is

$$
\diamond^{k}\left((-1)^{\mathrm{k}} \mathrm{R}_{2 \mathrm{k}}^{\mathrm{e}}(\mathrm{x}) * \mathrm{R}_{2 \mathrm{k}}^{\mathrm{H}}(\mathrm{x})\right)=\delta .
$$

In 2004, Yildirim, Sarikaya and Sermin $[7,8]$ first introduced the Bessel diamond operator $\diamond_{\mathrm{B}}^{\mathrm{k}}$ iterated $k$ times, and defined by

$$
\diamond_{\mathrm{B}}^{\mathrm{k}}=\left(\left(\sum_{i=1}^{p} \mathrm{~B}_{x_{i}}\right)^{2}-\left(\sum_{j=p+1}^{p+q} B_{x_{j}}\right)^{2}\right)^{k}
$$

where $B_{x_{i}}=\frac{\partial^{2}}{\partial x_{i}^{2}}+\frac{2 v_{i}}{x_{i}} \frac{\partial}{\partial x_{i}}, 2 v_{i}=2 \alpha_{i}+1, \alpha_{i}>-\frac{1}{2}, x_{i}>0$. The operator $\nabla_{B}^{k}$ can be expressed by $\diamond_{\mathrm{B}}^{\mathrm{k}}=\triangle_{\mathrm{B}}^{\mathrm{k}} \square_{\mathrm{B}}^{\mathrm{k}}=\square_{\mathrm{B}}^{\mathrm{k}} \triangle_{\mathrm{B}}^{\mathrm{k}}$, where

$$
\triangle_{B}^{k}=\left(\sum_{i=1}^{p} B_{x_{i}}\right)^{k} \text { and } \square_{B}^{k}=\left(\sum_{i=1}^{p} B_{x_{i}}-\sum_{j=p+1}^{p+q} B_{x_{j}}\right)^{k} .
$$

Yildirim, Sarikaya and Sermin $[7,8]$ have shown the convolution form $u(x)=(-1)^{k} S_{2 k}(x) * R_{2 k}(x)$ is a unique elementary solution of $\diamond_{\mathrm{B}}^{\mathrm{k}}$ that is

$$
\diamond_{\mathrm{B}}^{\mathrm{k}}\left((-1)^{\mathrm{k}} \mathrm{S}_{2 \mathrm{k}}(\mathrm{x}) * \mathrm{R}_{2 \mathrm{k}}(\mathrm{x})\right)=\delta,
$$

where $S_{2 k}(x)$ and $R_{2 k}(x)$ are defined by (2.5) and (2.6) with $\alpha=\gamma=2 k$, respectively. Next, Bunpog and Kananthai [1] have first introduced the operator $\left(\diamond_{B}+m^{4}\right)^{k}$ named Diamond Klien-Gordon Bessel operator iterated $k$ times and can be written in the following form

$$
\left(\diamond_{\mathrm{B}}+\mathrm{m}^{4}\right)^{\mathrm{k}}=\left(\left(\triangle_{\mathrm{B}}+\mathrm{m}^{2}\right)\left(\square_{\mathrm{B}}+\mathrm{m}^{2}\right)-\mathrm{m}^{2}\left(\triangle_{\mathrm{B}}+\square_{\mathrm{B}}\right)\right)^{\mathrm{k}},
$$

where $\square_{B}+m^{2}$ is the Bessel Klien-Gordon operator and $\triangle_{B}+m^{2}$ is the Bessel Helmholtz operator defined by

$$
\square_{B}+m^{2}=\sum_{i=1}^{p} B_{x_{i}}-\sum_{j=p+1}^{p+q} B_{x_{j}}+m^{2}
$$

and

$$
\triangle_{B}+m^{2}=\sum_{i=1}^{n} B_{x_{i}}+m^{2}
$$

The purpose of this work, first, we study the elementary solution or Green function of the $\left(\diamond_{B}+d^{2}\right)^{k}$, that is

$$
\left(\diamond_{\mathrm{B}}+\mathrm{d}^{2}\right)^{\mathrm{k}} \mathrm{G}(\mathrm{x})=\delta,
$$

where $G(x)$ is the Green function, $\delta$ is the Dirac delta distribution, $k$ is a nonnegative integer and $x=$ $\left(x_{1}, x_{2}, \cdots, x_{n}\right) \in \mathbb{R}^{n}$. We also consider the convolution of Green function. 
Finally, we are finding the solution of the equation

$$
\left(\nabla_{\mathrm{B}}+\mathrm{d}^{2}\right)^{\mathrm{k}} \mathrm{u}(\mathrm{x})=\sum_{\mathrm{r}=0}^{\mathrm{m}} \mathrm{c}_{\mathrm{r}}\left(\nabla_{\mathrm{B}}+\mathrm{d}^{2}\right)^{\mathrm{k}} \delta .
$$

We use the B-convolution for the generalized function. It was found that the type of the solution (1.4) that depend on the relationship between the values of $k$ and $m$ are as the following cases:

(1) If $m<k$ and $m=0$, then the solution of (1.4) is

$$
u(x)=c_{0} W_{2 k}(x),
$$

which is an elementary solution of the operator $\left(\diamond_{B}+d^{2}\right)^{k}$ in Theorem 3.1, is the ordinary function for $2 k \geqslant n+2|v|$, and is a tempered distribution for $2 k<n+2|v|$.

(2) If $0<m<k$, then the solution of (1.4) is

$$
u(x)=\sum_{r=1}^{m} c_{r} W_{2(k-r)}(x),
$$

which is an ordinary function for $2 k-2 r \geqslant n+2|v|$ and is tempered distribution for $2 k-2 r<$ $n+2|v|$.

(3) If $m \geqslant k$ and suppose $k \leqslant m \leqslant M$, then (1.4) has the solution

$$
u(x)=\sum_{m=k}^{M} c_{m}\left(\diamond_{B}+d^{2}\right)^{m-k} \delta,
$$

which is only the singular distribution.

Before proceeding that point, the following definitions and some important concepts are needed.

\section{Preliminaries}

Definition 2.1. Let $x=\left(x_{1}, x_{2}, \cdots, x_{n}\right)$ be a point of the $n$-dimensional Euclidean space $\mathbb{R}^{n}$ and write

$$
v=x_{1}^{2}+x_{2}^{2}+\cdots+x_{p}^{2}-x_{p+1}^{2}-x_{p+2}^{2}-\cdots-x_{p+q}^{2}
$$

where $p+q=n$ is the dimension of the space $\mathbb{R}^{n}$. Let $\Gamma_{+}=\left\{x \in \mathbb{R}^{n}: x_{1}>0\right.$ and $\left.u>0\right\}$ is the interior of forward cone and $\bar{\Gamma}_{+}$denotes it closure. For any complex number $\alpha$, define the function

$$
R_{\alpha}^{H}(v)= \begin{cases}\frac{v^{\frac{\alpha-n}{2}}}{K_{n}(\alpha)}, & \text { for } x \in \Gamma_{+}, \\ 0, & \text { for } x \notin \Gamma_{+},\end{cases}
$$

where the constant $K_{n}(\alpha)$ is given by the formula

$$
\mathrm{K}_{n}(\alpha)=\frac{\pi^{\frac{n-1}{2}} \Gamma\left(\frac{2+\alpha-n}{2}\right) \Gamma\left(\frac{1-\alpha}{2}\right) \Gamma(\alpha)}{\Gamma\left(\frac{2+\alpha-p}{2}\right) \Gamma\left(\frac{p-\alpha}{2}\right)} .
$$

The function $R_{\alpha}^{\mathrm{H}}(v)$ is called the Ultra-hyperbolic kernel of Marcel Riesz and was introduced by Nozaki [5]. 
It is well known that $R_{\alpha}^{H}(v)$ is an ordinary function if $\operatorname{Re}(\alpha) \geqslant n$ and is a distribution of $\alpha$ if $\operatorname{Re}(\alpha)<n$. Let supp $R_{\alpha}^{\mathrm{H}}(v)$ denote the support of $R_{\alpha}^{\mathrm{H}}(v)$ and suppose supp $R_{\alpha}^{\mathrm{H}}(v) \subset \bar{\Gamma}_{+}$, that is supp $R_{\alpha}^{\mathrm{H}}(v)$ is compact.

From Trione [6], $R_{2 k}^{H}(v)$ is an elementary solution of the operator $\square^{k}$, that is

$$
\square^{\mathrm{k}} \mathrm{R}_{2 \mathrm{k}}^{\mathrm{H}}(v)=\delta(x)
$$

Definition 2.2. Let $x=\left(x_{1}, x_{2}, \cdots, x_{n}\right)$ be a point of $\mathbb{R}^{n}$ and $|x|=\left(x_{1}^{2}+x_{2}^{2}+\cdots+x_{n}^{2}\right)^{\frac{1}{2}}$ the function $R_{\alpha}^{e}(x)$ denoted the elliptic kernel of Marcel Riesz and is defined by

$$
R_{\alpha}^{e}(x)=\frac{|x|^{\alpha-n}}{W_{n}(\alpha)}
$$

where

$$
W_{n}(\alpha)=\frac{\pi^{\frac{n}{2}} 2^{\alpha} \Gamma\left(\frac{\alpha}{2}\right)}{\Gamma\left(\frac{n-\alpha}{2}\right)}
$$

$\alpha$ is a complex parameter and $n$ is the dimension of $\mathbb{R}^{n}$.

Definition 2.3. Let $x=\left(x_{1}, x_{2}, \cdots, x_{n}\right), v=\left(v_{1}, v_{2}, \cdots, v_{n}\right) \in \mathbb{R}_{n}^{+}$. For any complex number $\alpha$, we define the distribution family $S_{\alpha}(x)$ by

$$
S_{\alpha}(x)=\frac{|x|^{\alpha-n-2|v|}}{w_{n}(\alpha)}
$$

where $|x|=x_{1}^{2}+x_{2}^{2}+\cdots+x_{n}^{2},|v|=v_{1}+v_{2}+\cdots+v_{n}$ and

$$
w_{n}(\alpha)=\frac{\prod_{i=1}^{n} 2^{v_{i}-\frac{1}{2}} \Gamma\left(v_{i}+\frac{1}{2}\right)}{2^{n+2|v|-2 \alpha} \Gamma\left(\frac{n+2|v|-\alpha}{2}\right)} .
$$

Definition 2.4. Let $x=\left(x_{1}, x_{2}, \cdots, x_{n}\right), v=\left(v_{1}, v_{2}, \cdots, v_{n}\right) \in \mathbb{R}_{n}^{+}$, and denote by

$$
\mathrm{V}=\mathrm{x}_{1}^{2}+\mathrm{x}_{2}^{2}+\cdots+\mathrm{x}_{\mathrm{p}}^{2}-\mathrm{x}_{\mathrm{p}+1}^{2}-\mathrm{x}_{\mathrm{p}+2}^{2}-\cdots-\mathrm{x}_{\mathrm{p}+\mathrm{q}}^{2}
$$

the nondegenerated quadratic form. Denote the interior of the forward cone by

$$
\Gamma_{+}=\left\{x \in \mathbb{R}_{n}^{+}: x_{1}>0, x_{2}>0, \cdots, x_{n}>0, V>0\right\},
$$

and $\bar{\Gamma}_{+}$denotes its closure. For any complex number $\gamma$ the distribution family $R_{\gamma}(x)$ is defined by

$$
R_{\gamma}(x)= \begin{cases}\frac{v^{\frac{\gamma-n-2|v|}{2}}}{K_{n}(\gamma)}, & \text { for } x \in \Gamma_{+}, \\ 0, & \text { for } x \notin \Gamma_{+}\end{cases}
$$

where

$$
\mathrm{K}_{\mathrm{n}}(\gamma)=\frac{\pi^{\frac{\mathrm{n}+2|v|-1}{2}} \Gamma\left(\frac{2+\gamma-\mathrm{n}-2|v|}{2}\right) \Gamma\left(\frac{1-\gamma}{2}\right) \Gamma(\gamma)}{\Gamma\left(\frac{2+\gamma-p-2|v|}{2}\right) \Gamma\left(\frac{p-\gamma}{2}\right)}
$$

where $\gamma$ is a complex number.

Definition 2.5. Let $x=\left(x_{1}, x_{2}, \cdots, x_{n}\right)$ be a point of $\mathbb{R}_{n}^{+}$, we define the function

$$
W_{\alpha}(x)=\sum_{r=0}^{\infty} \frac{(-1)^{r} \Gamma\left(\frac{\eta}{2}+r\right)}{r ! \Gamma\left(\frac{\eta}{2}\right)}\left(m^{2}\right)^{r}(-1)^{\frac{\alpha}{2}+r} S_{\alpha+2 r}(x) * R_{\alpha+2 r}(x),
$$

where the function $S_{\alpha+2 r}$ and $R_{\alpha+2 r}$ are defined by Definition 2.3 and Definition 2.4, respectively. 
Lemma 2.6. Let $\alpha$ and $\beta$ be complex numbers and $S_{\alpha}(x)$ be the function defined by (2.1). Then the following properties are valid

$$
\begin{aligned}
S_{0}(x) & =\delta(x), \\
S_{-2 k}(x) & =(-1)^{k} \triangle_{B}^{k} \delta, \\
\triangle_{B}^{k}\left\{S_{\alpha}(x)\right\} & =(-1)^{k} S_{\alpha-2 k}(x), \\
S_{\alpha}(x) * S_{\beta}(x) & =S_{\alpha+\beta}(x),
\end{aligned}
$$

where $\triangle_{\mathrm{B}}^{\mathrm{k}}$ is the Laplace Bessel operator iterated $\mathrm{k}$ times and defined by (1.2).

Proof. [4].

Lemma 2.7. Let $\alpha$ and $\beta$ be complex numbers and $R_{\gamma}(x)$ be the function defined by (2.2). Then the following properties are valid

$$
\begin{aligned}
\mathrm{R}_{0}(x) & =\delta(x), \\
\mathrm{R}_{-2 \mathrm{k}}(x) & =\square_{\mathrm{B}}^{\mathrm{k}} \delta, \\
\square_{\mathrm{B}}^{\mathrm{k}}\left\{\mathrm{S}_{\gamma}(x)\right\} & =\mathrm{S}_{\gamma-2 \mathrm{k}}(\mathrm{x}), \\
\mathrm{R}_{\alpha}(\mathrm{x}) * \mathrm{R}_{\beta}(x) & =\mathrm{R}_{\alpha+\beta}(x),
\end{aligned}
$$

where $\square_{\mathrm{B}}^{\mathrm{k}}$ is the Ultrahyperbolic Bessel operator iterated $\mathrm{k}$ times and defined by (1.2).

Proof. [4].

Lemma 2.8. The functions $S_{\alpha}(x)$ and $R_{\alpha}(x)$ defined by (2.1) and (2.2) respectively are homogeneous distribution of order $\alpha-n-2|v|$ and also tempered distribution.

Proof. Since $R_{\alpha}(x)$ and $S_{\alpha}(x)$ satisfy the Euler equation, that is

$$
(\alpha-n-2|v|) R_{\alpha}(x)=\sum_{i=1}^{n} x_{i} \frac{\partial}{\partial x_{i}} R_{\alpha}(x),
$$

and

$$
(\alpha-n-2|v|) S_{\alpha}(x)=\sum_{i=1}^{n} x_{i} \frac{\partial}{\partial x_{i}} S_{\alpha}(x) .
$$

We have $R_{\alpha}(x)$ and $S_{\alpha}(x)$ as homogeneous distributions of order $\alpha-n-2|v|$ and Donoghue [2] has proved that every homogeneous distribution is a tempered distribution. That completes the proof.

Lemma 2.9 (The convolution of tempered distribution). The convolution $R_{\alpha}(x) * S_{\alpha}(x)$ exists and is a tempered distribution.

Proof. Choosing supp $R_{\alpha}(x)=K \subset \Gamma_{+}$where $K$ is a compact set, the function $R_{\alpha}(x)$ is a tempered distribution with compact support and by Donoghue [2] $\mathrm{R}_{\alpha}(x) * S_{\alpha}(x)$ exists and is a tempered distribution.

Lemma 2.10. Given the equation $\diamond_{\mathrm{B}}^{\mathrm{k}} u(x)=\delta(x)$ for $x \in \mathbb{R}_{n}^{+}$, where $\diamond_{\mathrm{B}}^{\mathrm{k}}$ defined by (1.1), and

$$
\mathrm{u}(\mathrm{x})=(-1)^{\mathrm{k}} \mathrm{S}_{2 \mathrm{k}}(\mathrm{x}) * \mathrm{R}_{2 \mathrm{k}}(x),
$$

where $S_{2 k}(x)$ and $R_{2 k}(x)$ are defined by (2.1) and (2.3) with $\alpha=2 k, \gamma=2 k$, respectively. We obtain $(-1)^{k} S_{2 k}(x) *$ $\mathrm{R}_{2 \mathrm{k}}(\mathrm{x})$ is an elementary solution of the operator $\diamond_{\mathrm{B}}^{\mathrm{k}}$. That is,

$$
\diamond_{B}^{k}\left((-1)^{k} S_{2 k}(x) * R_{2 k}(x)\right)=\delta(x) .
$$

Proof. $[7,8]$. 
Lemma 2.11. Let $\alpha$ and $\beta$ be complex numbers. The following formulas are valid

$$
\begin{aligned}
W_{0}(x) & =\delta(x), \\
W_{\alpha} * W_{\beta} & =W_{\alpha+\beta}, \\
W_{\alpha} * W_{-2 k} & =W_{\alpha-2 k} .
\end{aligned}
$$

Proof. By Definition 2.3, we obtain

$$
W_{0}(x)=\delta(x)
$$

By Definition 2.3 again, we have

$$
\begin{aligned}
W_{\alpha}(x) * W_{\beta}(x)= & \sum_{r=0}^{\infty}\left(\begin{array}{c}
-\frac{\alpha}{2} \\
r
\end{array}\right)\left(m^{2}\right)^{r}(-1)^{\frac{\alpha}{2}+r} S_{\alpha+2 r}(x) * R_{\alpha+2 r}(x) . \\
& * \sum_{s=0}^{\infty}\left(\begin{array}{c}
-\frac{\beta}{2} \\
s
\end{array}\right)\left(m^{2}\right)^{s}(-1)^{\frac{\beta}{2}+s} S_{\beta+2 s}(x) * R_{\beta+2 s}(x) \\
= & \sum_{r=0}^{\infty} \sum_{s=0}^{\infty}\left(\begin{array}{c}
-\frac{\alpha}{2} \\
r
\end{array}\right)\left(\begin{array}{c}
-\frac{\beta}{2} \\
s
\end{array}\right)\left(m^{2}\right)^{r+s}(-1)^{\frac{\alpha+\beta}{2}+r+s} \\
& \times\left(S_{\alpha+2 r}(x) * R_{\alpha+2 r}(x)\right) *\left(S_{\beta+2 s}(x) * R_{\beta+2 s}(x)\right) \\
= & \sum_{r=0}^{\infty} \sum_{s=0}^{\infty}\left(\begin{array}{c}
-\frac{\alpha}{2} \\
r
\end{array}\right)\left(\begin{array}{c}
-\frac{\beta}{2} \\
s
\end{array}\right)\left(m^{2}\right)^{r+s}(-1)^{\frac{\alpha+\beta}{2}+r+s}\left(S_{\alpha+\beta+2(r+s)}(x) * R_{\alpha+\beta+2(r+s)}(x)\right) \\
= & \sum_{k=0}^{\infty}\left(m^{2}\right)^{k}\left[\begin{array}{c}
k \\
r=0
\end{array}\left(\begin{array}{c}
-\frac{\alpha}{2} \\
r
\end{array}\right)\left(\begin{array}{c}
-\frac{\beta}{2} \\
k-r
\end{array}\right)\right](-1)^{\frac{\alpha+\beta}{2}+k}\left(S_{\alpha+\beta+2 k}(x) * R_{\alpha+\beta+2 k}(x)\right) .
\end{aligned}
$$

By properties

$$
\sum_{r=0}^{k}\left(\begin{array}{c}
-\frac{\alpha}{2} \\
r
\end{array}\right)\left(\begin{array}{c}
-\frac{\beta}{2} \\
k-r
\end{array}\right)=\left(\begin{array}{c}
-\frac{\alpha+\beta}{2} \\
k
\end{array}\right) .
$$

The Equation (2.8) becomes

$$
\begin{aligned}
W_{\alpha}(x) * W_{\beta}(x) & =\sum_{r=0}^{\infty}\left(\begin{array}{c}
-\frac{\alpha+\beta}{2} \\
r
\end{array}\right)\left(m^{2}\right)^{r}(-1)^{\frac{\alpha+\beta}{2}+k} S_{\alpha+\beta+2 k}(x) * R_{\alpha+\beta+2 k}(x) \\
& =W_{\alpha+\beta}(x) .
\end{aligned}
$$

Thus,

$$
W_{\alpha}(x) * W_{\beta}(x)=W_{\alpha+\beta}(x)
$$

Putting $\beta=-2 k$ in (2.9), we obtain

$$
\mathrm{W}_{\alpha}(x) * \mathrm{~W}_{-2 \mathrm{k}}(x)=\mathrm{W}_{\alpha-2 \mathrm{k}}(x)
$$

That completes the proof.

\section{Main results}

Theorem 3.1. Given the equation

$$
\left(\diamond_{B}+d^{2}\right)^{k} u(x)=\delta(x),
$$

for $x \in \mathbb{R}_{n}^{+}$and $\left(\diamond_{\mathrm{B}}+\mathrm{d}^{2}\right)^{\mathrm{k}}$ is the Diamond Klein Gordon operator iterated $\mathrm{k}$ times defined by (1.3), we obtain

$$
u(x)=W_{2 k}(x)
$$


is an elementary solution or Green function of the operator $\left(\nabla_{\mathrm{B}}+\mathrm{d}^{2}\right)^{\mathrm{k}}$ and $\mathrm{W}_{2 \mathrm{k}}(\mathrm{x})$ is defined by $(2.7)$ with $\alpha=2 \mathrm{k}$. The function $\mathrm{W}_{2 \mathrm{k}}(\mathrm{x})$ has the following properties

$$
W_{0}(x)=\delta(x)
$$

and

$$
\left(\diamond_{B}+d^{2}\right)^{k}\left\{W_{\alpha}(x)\right\}=W_{\alpha-2 k}(x)
$$

Proof. In fact,

$$
\left(\diamond_{\mathrm{B}}+\mathrm{d}^{2}\right)^{-\frac{\alpha}{2}}=\left\{\diamond_{\mathrm{B}}\left(1+\mathrm{d}^{2} \diamond_{\mathrm{B}}^{-1}\right)\right\}^{-\frac{\alpha}{2}}=\diamond_{\mathrm{B}}^{-\frac{\alpha}{2}}\left(1+\mathrm{d}^{2} \diamond_{\mathrm{B}}^{-1}\right)^{-\frac{\alpha}{2}}
$$

and

$$
\begin{aligned}
\left(1+d^{2} \diamond_{B}^{-1}\right)^{-\frac{\alpha}{2}} \delta & =\sum_{r=0}^{\infty}\left(\begin{array}{c}
-\frac{\alpha}{2} \\
r
\end{array}\right)\left(d^{2} \diamond_{B}^{-1}\right)^{r} \delta \\
& =\sum_{r=0}^{\infty}\left(\begin{array}{c}
-\frac{\alpha}{2} \\
r
\end{array}\right) d^{2 r} \diamond_{B}^{-r} \delta
\end{aligned}
$$

Thus,

$$
\begin{aligned}
\diamond_{\mathrm{B}}^{-\frac{\alpha}{2}}\left(1+\mathrm{d}^{2} \diamond_{\mathrm{B}}^{-1}\right)^{-\frac{\alpha}{2}} & =\diamond^{-\frac{\alpha}{2}} \sum_{\mathrm{r}=0}^{\infty}\left(\begin{array}{c}
-\frac{\alpha}{2} \\
\mathrm{r}
\end{array}\right)\left(\mathrm{d}^{2} \diamond_{\mathrm{B}}^{-1}\right)^{\mathrm{r}} \delta \\
& =\sum_{\mathrm{r}=0}^{\infty}\left(\begin{array}{c}
-\frac{\alpha}{2} \\
r
\end{array}\right) \mathrm{d}^{2 \mathrm{r}} \diamond_{\mathrm{B}}^{-\frac{\alpha}{2}-\mathrm{r}} \delta .
\end{aligned}
$$

From the above equation, we get

$$
\begin{aligned}
\left(\diamond_{\mathrm{B}}+\mathrm{d}_{\mathrm{B}}^{2}\right)^{-\frac{\alpha}{2}} \delta & =\sum_{\mathrm{r}=0}^{\infty}\left(\begin{array}{c}
-\frac{\alpha}{2} \\
r
\end{array}\right) \mathrm{d}^{2 \mathrm{r}} \diamond_{\mathrm{B}}^{-\frac{\alpha}{2}-\mathrm{r}} \delta \\
& =\sum_{\mathrm{r}=0}^{\infty}\left(\begin{array}{c}
-\frac{\alpha}{2} \\
r
\end{array}\right) \mathrm{d}^{2 \mathrm{r}} \triangle_{\mathrm{B}}^{-\frac{\alpha}{2}-\mathrm{r}} \square_{\mathrm{B}}^{-\frac{\alpha}{2}-\mathrm{r}} \delta \\
& =\sum_{r=0}^{\infty}\left(\begin{array}{c}
-\frac{\alpha}{2} \\
r
\end{array}\right) \mathrm{d}^{2 \mathrm{r}}(-1)^{\frac{\alpha}{2}+\mathrm{r}} S_{2\left(\frac{\alpha}{2}+r\right)}(x) * R_{2\left(\frac{\alpha}{2}+r\right)}(v) \\
& =\sum_{r=0}^{\infty}\left(\begin{array}{c}
-\frac{\alpha}{2} \\
r
\end{array}\right) \mathrm{d}^{2 r}(-1)^{\frac{\alpha}{2}+r} S_{\alpha+2 r}(x) * R_{\alpha+2 r}(x) \\
& =W_{\alpha}(x) .
\end{aligned}
$$

If we put $\alpha=-2 k$, we obtain

$$
\left(\diamond_{B}+d^{2}\right)^{k} \delta=W_{-2 k}(x) .
$$

Putting $k=0$ in (3.2), we obtain

$$
W_{0}(x)=\delta(x)
$$

By Lemma 2.6, we have

$$
W_{\alpha}(x) * W_{\beta}(x)=W_{\alpha+\beta}(x)
$$

Putting $\beta=-2 k$, we obtain

$$
\begin{aligned}
W_{\alpha}(x) * W_{-2 k}(x) & =W_{\alpha-2 k}(x), \\
W_{\alpha}(x) *\left(\diamond+d^{2}\right)^{k} \delta & =W_{\alpha-2 k}(x)
\end{aligned}
$$




$$
\left(\diamond_{\mathrm{B}}+\mathrm{d}^{2}\right)^{\mathrm{k}} \mathrm{W}_{\alpha}(\mathrm{x}) * \delta=\mathrm{W}_{\alpha-2 \mathrm{k}}(\mathrm{x}) .
$$

If we put $\alpha=2 k$ in (3.3), we obtain

$$
\left(\diamond+d^{2}\right)^{k} \delta * W_{2 k}(x)=W_{0}(x)=\delta(x) .
$$

It follows that $W_{2 k}(x)$ is an elementary solution or Green function of the operator $\left(\diamond+d^{2}\right)^{k}$. That completes the proof.

Theorem 3.2. For $0<\mathrm{r}<\mathrm{k}$

$$
\left(\diamond_{B}+d^{2}\right)^{r} W_{2 k}(x)=W_{2(k-r)}(x)
$$

and for $\mathrm{k} \leqslant \mathrm{m}$

$$
\left(\diamond_{B}+d^{2}\right)^{m}=\left(\diamond_{B}+d^{2}\right)^{m-k} \delta,
$$

where $\left(\nabla_{\mathrm{B}}+\mathrm{d}^{2}\right)^{\mathrm{k}}$ is the Diamond Bessel Klein Gordon operator iterated $\mathrm{k}$ times defined by (1.3), $\delta$ is the Dirac delta distribution and the function $\mathrm{W}_{2 \mathrm{k}}(\mathrm{x})$ defined by (2.7) with $\alpha=2 \mathrm{k}$.

Proof. For $0<\mathrm{r}<\mathrm{k}$, from Theorem 3.1,

$$
\left.\left(\diamond_{\mathrm{B}}+\mathrm{d}^{2}\right)^{\mathrm{k}} \mathrm{W}_{2 \mathrm{k}}(\mathrm{x})\right)=\delta .
$$

We can write the above equation in the following form

$$
\left(\nabla_{\mathrm{B}}+\mathrm{d}^{2}\right)^{\mathrm{k}-\mathrm{r}}\left(\nabla_{\mathrm{B}}+\mathrm{d}^{2}\right)^{\mathrm{r}} \mathrm{W}_{2 \mathrm{k}}(\mathrm{x})=\delta,
$$

or

$$
\left(\diamond_{B}+d^{2}\right)^{k-r} \delta *\left(\diamond_{B}+d^{2}\right)^{r} W_{2 k}(x)=\delta .
$$

We have used the convolution of both sides by $W_{2(k-r)}(x)$, we obtain

$$
W_{2(k-r)} *\left(\diamond_{B}+d^{2}\right)^{k-r} \delta *\left(\diamond_{B}+d^{2}\right)^{r} W_{2 k}(x)=W_{2(k-r)}(x) * \delta .
$$

By property of convolution, we have

$$
\left(\diamond_{B}+d^{2}\right)^{k-r} W_{2(k-r)} *\left(\diamond_{B}+d^{2}\right)^{r} W_{2(k)}(x)=W_{2(k-r)}(x) .
$$

By Lemma 2.7, we obtain

$$
\delta *\left(\diamond_{\mathrm{B}}+\mathrm{d}^{2}\right)^{\mathrm{r}} \mathrm{W}_{2 \mathrm{k}}(\mathrm{x})=\mathrm{W}_{2(\mathrm{k}-\mathrm{r})}(\mathrm{x})
$$

or

$$
\left(\diamond_{B}+d^{2}\right)^{r} W_{2 k}(x)=W_{2(k-r)}(x)
$$

as required. For $k \leqslant m$

$$
\begin{aligned}
\left.\left(\diamond_{B}+d^{2}\right)^{m} W_{2 k}(x)\right) & \left.=\left(\diamond_{B}+d^{2}\right)^{m-k}\left(\diamond_{B}+d^{2}\right)^{k} W_{2 k}(x)\right) \\
& =\left(\diamond_{B}+d^{2}\right)^{m-k} \delta .
\end{aligned}
$$

It follows that

$$
\left.\left(\nabla_{B}+d^{2}\right)^{m} W_{2 k}(x)\right)=\left(\diamond_{B}+d^{2}\right)^{m-k} \delta .
$$

That completes the proof. 
Theorem 3.3. Given the linear differential equation

$$
\left(\nabla_{B}+d^{2}\right)^{k} u(x)=\sum_{r=0}^{m} c_{r}\left(\nabla_{B}+d^{2}\right)^{k} \delta .
$$

Then the type of solution (3.4) that depend on the relationship between the values of $\mathrm{k}$ and $\mathrm{m}$ are as the following cases:

(1) if $\mathrm{m}<\mathrm{k}$ and $\mathrm{m}=0$, then the solution of (3.4) is

$$
\left.u(x)=c_{0} W_{2 k}(x)\right),
$$

which is an elementary solution of the $\left(\diamond_{\mathrm{B}}+\mathrm{d}^{2}\right)^{\mathrm{m}}$ operator in Theorem 3.1;

(2) if $0<m<k$, then the solution of (3.4) is

$$
u(x)=\sum_{r=1}^{m} c_{r} W_{2(k-r)}(x),
$$

which is an ordinary function for $2 k-2 r \geqslant n+2|v|$ and is tempered distribution for $2 k-2 r<n+2|v|$;

(3) if $m \geqslant k$ and suppose $k \leqslant m \leqslant M$, then (3.4) has the solution

$$
u(x)=\sum_{r=k}^{M} c_{r}\left(\diamond_{B}+d^{2}\right)^{r-k} \delta,
$$

which is only the singular distribution.

Proof.

(1) For $\mathrm{m}=0$, we have $\left(\diamond_{\mathrm{B}}+\mathrm{d}^{2}\right)^{\mathrm{k}} \mathrm{u}(\mathrm{x})=\mathrm{c}_{0} \delta$, and by Theorem 3.1 we obtain

$$
u(x)=W_{2 k}(x) \text {. }
$$

Now, $W_{2 k}(x)$ analytic function for $2 k \geqslant n+2|v|$ and also $W_{2 k}(x)$ exists and is an analytic function by (3.1). It follows that $W_{2 k}(x)$ is an ordinary function for $2 k \geqslant n+2|v|$ and is a tempered distribution with $2 k<n+2|v|$.

(2) For the case $0<m<k$, we have

$$
\begin{aligned}
\left(\nabla_{B}+d^{2}\right)^{k} u(x) & =\sum_{r=1}^{m} c_{r}\left(\diamond_{B}+d^{2}\right)^{r} \delta, \\
& =c_{1}\left(\diamond_{B}+d^{2}\right) \delta+c_{2}\left(\diamond_{B}+d^{2}\right)^{2} \delta+\cdots+c_{m}\left(\diamond_{B}+d^{2}\right)^{k} \delta .
\end{aligned}
$$

Convolving both sides of the above equation by $W_{2 k}(x)$, we obtain

$$
\begin{aligned}
W_{2 k}(x) *\left(\diamond_{B}+d^{2}\right)^{k} u(x)= & c_{1} W_{2 k}(x)\left(\diamond_{B}+d^{2}\right) \delta+c_{2} W_{2 k}(x)\left(\diamond_{B}+d^{2}\right)^{2} \delta \\
& +\cdots+c_{m} W_{2 k}(x)\left(\diamond_{B}+d^{2}\right)^{k} \delta \\
u(x) *\left(\diamond_{B}+d^{2}\right)^{k} W_{2 k}(x)= & c_{1}\left(\diamond_{B}+d^{2}\right) W_{2 k}(x)+c_{2}\left(\diamond_{B}+d^{2}\right)^{2} W_{2 k}(x) \\
& +\cdots+c_{m}\left(\diamond_{B}+d^{2}\right)^{m} W_{2 k}(x),
\end{aligned}
$$




$$
\begin{aligned}
u(x)= & c_{1}\left(\nabla_{B}+d^{2}\right) W_{2 k}(x)+c_{2}\left(\nabla_{B}+d^{2}\right)^{2} W_{2 k}(x) \\
& +\cdots+c_{m}\left(\nabla_{B}+d^{2}\right)^{m} W_{2 k}(x) .
\end{aligned}
$$

By Theorem 3.1 and Theorem 3.2, we obtain

$$
u(x)=c_{1} W_{2(k-1)}(x)+c_{2} W_{2(k-2)}(x)+\cdots+c_{m} W_{2(k-m)}(x),
$$

or

$$
u(x)=\sum_{r=1}^{m} c_{r} W_{2(k-r)}(x) .
$$

Similarly, as in the case (1), $u(x)$ is an ordinary function for $2 k-2 r \geqslant n+2|v|$ and is a tempered distribution for $2 k-2 r<n+2|v|$.

(3) For the case $m \geqslant k$ and suppose $k \leqslant m \leqslant M$, we have

$$
\left(\nabla_{\mathrm{B}}+\mathrm{d}^{4}\right) \mathrm{u}(\mathrm{x})=\mathrm{c}_{\mathrm{k}}\left(\nabla_{\mathrm{B}}+\mathrm{d}^{4}\right)^{\mathrm{k}} \delta+\mathrm{c}_{\mathrm{k}+1}\left(\nabla_{\mathrm{B}}+\mathrm{d}^{2}\right)^{\mathrm{k}+1} \delta+\cdots+\mathrm{c}_{M}\left(\diamond_{\mathrm{B}}+\mathrm{d}^{2}\right)_{\mathrm{B}}^{\mathrm{M}} \delta
$$

We convolved both sides of the above equation by $W_{2 k}(x)$, we obtain

$$
\begin{aligned}
W_{2 k}(x) *\left(\nabla_{B}+d^{2}\right)^{k} u(x)= & c_{1} W_{2 k}(x)\left(\nabla_{B}+d^{2}\right) \delta+c_{2} W_{2 k}(x)\left(\nabla_{B}+d^{2}\right)^{2} \delta \\
& +\cdots+c_{m} W_{2 k}(x)\left(\diamond_{B}+d^{2}\right)^{k} \delta .
\end{aligned}
$$

By Theorem 3.1 and Theorem 3.2 again, we obtain

$$
\begin{aligned}
u(x) & =c_{k} \delta+c_{k+1}\left(\nabla_{B}+d^{2}\right) \delta+c_{k+2}\left(\nabla_{B}+d^{2}\right)^{2} \delta+\cdots+c_{M}\left(\nabla_{B}+d^{2}\right)^{M-k} \delta \\
& =\sum_{m=k}^{M} c_{m}\left(\diamond_{B}+d^{2}\right)^{m-k} \delta .
\end{aligned}
$$

Since $\left(\diamond_{B}+d^{2}\right)^{m-k} \delta$ is a singular distribution, hence $u(x)$ is only the singular distribution. That completes the proofs.

\section{Acknowledgment}

The authors would like to thank The Thailand Research Fund, The Commission Higher Education and Graduate school, Maejo University, Thailand for financial support and also Prof. Amnuay Kananthai Department of Mathematics, Chiang Mai University for the helpful discussion.

\section{References}

[1] C. Bunpog, A. Kananthai, On the Bessel Diamond operator, J. Appl. Functional Anal., (2009), 10-19. 1

[2] W. F. Donoghue, Distributions and Fourier Transform, Academic Press, New York, (1969). 2, 2

[3] A. Kananthai, On the Solution of the n-Dimensional Diamond Operator, Appl. Math. Comput., 88 (1997), 27-37. 1, 1

[4] A. Manuel, T. Aguirre, Some properties of Bessel Elliptic kernel and Bessel ultra-hyperbolic kernel, Thai J. Math., 6 (2008), 171-190. 2, 2

[5] Y. Nozaki, On Riemann-Liouville integral of Ultra-hyperbolic type, Kodai Math. Sem. Rep., 16 (1964), 69-87. 2.1

[6] S. E. Trione, On Marcel Riesz's ultrahyperbolic kernel, Stud. Appl. Math., 79 (1988), 185-191. 2

[7] H. Yildirim, Riesz potentials generated by a generalized shift operator, Ph.D. Thesis, Ankara University, (1995). 1, 2

[8] H. Yildirim, M. Z. Sarikaya, S. Öztürk, The solution of the n-dimensional Bessel diamond operator and the Fourier-Bessel transform of their convolution, Proc. Indian Acad. Sci. Math. Sci., 114 (2004), 375-387. 1, 2 